\title{
LANCASHIRE SECTION: Meeting at Stockport, 13th March, 1919: Mr. J. Higson in the Chair: DOUBLING
}

\section{J. Winterbottom M.sc.Tech.}

To cite this article: J. Winterbottom M.sc.Tech. (1919) LANCASHIRE SECTION: Meeting at Stockport, 13th March, 1919: Mr. J. Higson in the Chair: DOUBLING, Journal of the Textile Institute Proceedings and Abstracts, 10:7, 188-190, DOI: 10.1080/00405001908630913

To link to this article: http://dx.doi.org/10.1080/00405001908630913

\section{曲 Published online: 24 Nov 2008.}

\section{Submit your article to this journal 지}

Џ Article views: 3 
ROLLER GIN.

Seed Cotton (Kapas) 20,750 lbs.

\begin{tabular}{|c|c|c|c|}
\hline Lint produced & $\begin{array}{l}\text { Lbs. } \\
6,815\end{array}$ &.. & $\begin{array}{l}\text { Cent. } \\
32 \cdot 843\end{array}$ \\
\hline Seeds produced & 13,753 & .. & $66 \cdot 278$ \\
\hline & nil & .. & \\
\hline Invisible loss $\ldots \ldots \ldots$. & 182 & .. & 0.879 \\
\hline Blow Room Droppings & 447 & .. & $6 \cdot 559$ \\
\hline Invisible $\operatorname{loss}_{\ldots} \ldots \ldots \ldots \ldots \ldots$ & 42 & .. & $0 \cdot 616$ \\
\hline Card strips and fly $\ldots \ldots \ldots \ldots$ & 392 & .. & $5 \cdot 752$ \\
\hline $\begin{array}{l}\text { Good waste in Blow Room } \\
\text { Good waste in Card Room and }\end{array}$ & 49 & . & $0 \cdot 719$ \\
\hline Spinning Room $\ldots \ldots \ldots \ldots$ & 200 & .. & $2 \cdot 934$ \\
\hline Bondas $\ldots \ldots \ldots \ldots \ldots$ & 100 & .. & $1 \cdot 467$ \\
\hline Oily cotton.... & 65 & .. & 0.953 \\
\hline Reeling waste .... & 47 & .. & 0.689 \\
\hline Invisible loss. & 129 & $\ldots$ & $1.892>>$ \\
\hline
\end{tabular}
lbs.

Yarn produced 5,344 lbs.; waste produced 1,471

Wrapping counts : Test on one lea (=120 yards) :-

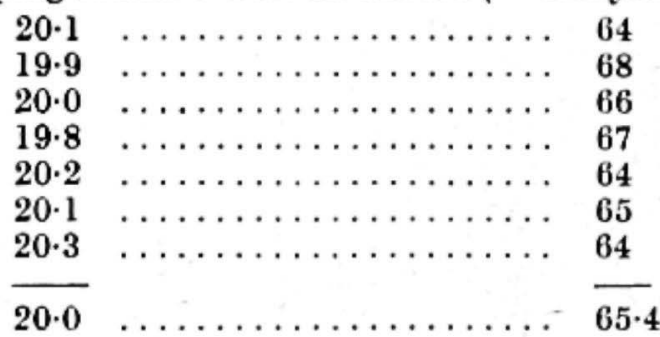

LANCASHIRE SECTION

Meeting at Stockport, 13th March, 1919.

Mr. J. Hrason in the Chair.

\section{DOUBLING}

By J. Winterbottom, M.Sc.Tech.

THE uncertainty of the behaviour of single yarns, in the process of doubling, forms a fitting subject for consideration and discussion at Stockport. The subject appeals to the interest of both spinner and doubler-to the spinner because it has to do with the outstanding and necessary qualities of his finished product for doubling, and to the doubler beceuse his raw material, and its development in the course of doubling, is involved. The essentials in single yarn for doubling, and the developments and re-ections during doubling, are matters which demand attention.

It is of fundamental importance to have in mind exactly what takes place in the body or mass of fibres during spinning. The object of the twist is understood as being to compact the fibres about their common axis ; to bind them and secure cohesion, the degree of cohesion being dependent upon the amount of the twist applied and the length of the fibres. This is the common service rendered by twist, but it is elso used for other purposes, such as creating hard effects, imparting shrinking and elastic properties, and crinkling effects, as in crepe yarn. For ordinary purposessetting aside for the moment the observance of the various twist standards or co-efficients-the purpose of twist may be said to be fully attained immediately it imparts that cohesion amongst the fibres which establishes them so that they do not slip under the tensile strain which yarns are required to resist. To carry twisting beyond this point has an adverse effect upon the strength.

The next point to have in mind is the effect which increasing twist exerts upon the measure of length occupied by the fibres in the single yarn, measured upon the same plane as the axis. From being straight as they emerge from the rollers, the fibres assume a spiral order-hence their contraction as the amount of twist is increased. Contraction increases with each added twist. Besides the spiral order here noted, each fibre is twisted upon its axis individually, which is proved in many ways. The effect of this, also, must cause each fibre to diminish in length. Further, the action hardens the fibres. What really occurs, then, is : that by twisting a body of fibres in this state the fibres are twisted individually and at the same time laid spirally, as the twisting proceeds. Thus, when they are subjected to tension, part of the tension force is converted into a shearing force. It is well known that all cellular matter is weakened by torque or twist, from like cause. This is evident in that by twist alone it is possible to break or shear such matter. The contraction in question is further augmented by the application of tension to the extent which the fibres tend to slide upon each other. The general conclusion reached, therefore, is that the yarn contracts in proportion to the degree of spirality developed by the fibres after emerging from the rollers in the straight state.

What occurs if we untwist yarn? The answer to this question is highly important. It is important that one should clearly realise what occurs. The spini ning twist causes the fibres to cohere. The fibres compact, and as they are twisted axially and individually they are compressed, as well as contracted. The fibres are also shortened, when measured in the plane of their axis. Upon untwisting single yarn, it is, therefore quite obvious that the contrary occurs. This raises a further point for consideration and that is : What takes place when two or more such yarns are placed together and twisted in the reverse direction to the twist in the singles? In doubling, this is the common course adopted in preparing almost all except the "strength" types of yarn. In the latter, twisting in the same direction as the single is often necessary. This, obviously, creates conditions which will require separate consideration. When the twist is in the reverse direction to the singles, we have to consider how this operates upon the fibres individually, as well as upon the two or several singles individually and collectively. Taking these in the order stated, we have the fibres tending to expand and extend individually by reason of the extraction of their twist. At the same time, the whole of the fibres comprised in each single are tending to expand collectively as a result of the diminution of spirality. These changes are occurring as the two or several singles are assuming the developments previously described. We have, then, the following conditions operating at the same time :-(1) Extending and expanding of fibres individually, due to the extraction of individual twist ; (2) extending and expanding of fibres individually, due to change in their spiral order; (3) contraction of the singles, relative to their common centre, on account of their assuming a spiral order common to the several singles.

The last-named condition, being opposite in its effects, will, to a greater or less extent, absorb the 
extension and expansion named in the items (1) and (2); it will exceed them when the singles are only slightly twisted; it will incompletely absorb them when the singles are twisted considerably. The point to be realised is that at one point only, between these two extreme twists, will the extensions and expansions of the fibres and the singles be just balanced by the opposite actions of doubling. This furnishes the explanation of why we get different lengths of folded yarn in proportion to the singles user in doubling, some yielding a length of folded yorn in excess of and others a length less than that of the singles; also, why, in doubling, yarns compact so differently, and hence their variations in appearance, mainly due to the order and compactness of the fibres and the twist markings or spiral order of the several bodies of yarn of which the doubled yarn is composed. When the folding twist is in the same direction as that of the singles, the following conditions are set up :(1) The fibres continue to contract in diameter and in length, due to twist in the individual fibres and the spiral order; (2) the two or several singles continue to contract in diameter and in length, due also to their spiral order, this latter condition occurring individually by reason of the spirality increasing with the twist inserted in common; (3) contraction of the two or several singles-as to length and diameter-as they are being placed spirally about each other, producing a state the opposite of enfolding; hence, instead of being drawn to a common centre, each is buckling and hardening, so that in place of a balanced effect being gradually approached the opposite is produced. The yarn, therefore, becomes very difficult to control and crinkles, becoming brittle and very dark in shade. The crinkling and darkening of shade develop very rapidly, and accountably so in view of what is taking place. Here, each fold-twist has a greater value than the number of the fold times the terminal twist put in each of the singles. And, as the twist proceeds, this value is constantly increasing. This consideration is useful when the conditions already named are sought, and also when the final thread, similarly composed, demands abnormal contraction in the final doubling stage. This is the case, at any rate, in certain of the strength types and in others which require to be smooth on the exterior and to be highly compacted. In these strength types, such smooth and compact state is reached more expeditiously than by the form of twiat indicated by putting in the preparation twist in the reverse order to that of the singles. Besides, it gives a measure of compactness hardly attainable otherwise.

Sufficient has now been said of the developments in the course of doubling to enable certain deductions to be made as to the effects which they produce; also, the conditions which must be fulfilled in order to produce the many typical characteristics aimed at in doubling. Briefly summarised, the aims of doubling, exclusive of specialities, may be stated as follows :-

(1) To provide means whereby two or more singles or other threads are built up spirally, simple or com. pound, in such a way that they become interlocked, the individual twist in the fibres being augmented or extracted by the identical or counteracting twist respectively. It is, therefore, a way of building up two or more yarns, in the course of which the initial twist is extracted or augmented as required. Hence, it is possible to make folded yarn with the twist in its fibres reduced to zero or any other predetermined measure.

(2) To stabilise twist and strength.

(3) To obtain a greater measure of strength than is procurable in single yarn or like count.

(4) To obtain more uniform yarn; softer or smoother effects; or certain surface markings.

(5) To obtain a smaller thread or a sharper definition than is procurable in a like count of single.

(6) To obtain some combination of two or more of the characteristics named.

Twist is stabilised or fixed by the extraction of the twist in the fibres and singles to an extent corres. ponding to the fold twist. The higher torque value of the same, per turn of twist, accumulating with the amount, counterbalances that remaining in the fibres and singles. At a certain point, equilibrium is reached and is evident when the yarn has no inclination to turn axially when free.

Strength is stabilised by the fixation of the twist.

The greater measure of strength of twofolds as compared with singles of like count arises from the less twist in the fibres accompanied with a uniting or compressing force, in no way inferior to that originally obtained by twist, but now replaced by the interlocking formation of the bands of fibres.

The uniforming effect arises from the combination; softening from the extraction of twist and the withdrawal of its hardening influence. At the same time, the rolling action, one thread upon another, coupled with the laying force caused by their rolling upon the surfaces with which they are in contact, lay the fibres and makes smooth their surface.

The surface markings are natural markings due to the bodies in folding remaining more or less distinct. This is intensified with the measure of twist in the single-that is, it becomes more pronounced with increasing single twist. The smaller thread is due to the greater compactness consequent on the extent to which the contraction of the two or more singles, in common, exceeds the expansion and extension of the singles as the twisting proceeds. This is determined by the measure of twist in the original singles, coupled with the tension applied in doubling.

We may at this point consider some of the develop. ments of a defective character. When twist is applied to two or more singles which differ at any identical point-in substance, texture, size, or twist-then they do not behave similarly. If they are unlike in twist only, this will cause extension and expansion to be dissimilar - the one having the most twist extending and expanding at a greater rate than the other, and thus causing a pronounced spiral effect. If they are alike except in size, a somewhat similar effect is produced, contrast in size being the special feature in this case. If they are alike except in regard to tension, then the spiral state is less pronounced. This is caused by a slight projection of the thread affected. When all are alike, except as to texture, the softer threads. conform more readily to the haraher threads, thereby causing a spiral state which is only slight. All single 
yarns vary in size, twist, and strength. The variations present are in a measure increasing in nature and amount with the lowering standard of quality, and they cause the chief defects which arise in doubling. It is on account of the abnormal extent of the development of these " single " faults in the process of doubling, that those understanding the work are so particular in the selection of their yarn. They know that the product will be unsatisfactory and that the wastage and trouble will be serious if unstable yarn is used, and it would be commercially impossible to proceed under such conditions. When the corresponding portions of each of the threads folderl vary, then the parts do not conform regularly with each other, but cause irregular spiral markings, bulging surface, deficient tensile resistance and elasticity, breakages and interruptions, the measure of these defects being in accordance with the varying state and developing far beyond their apparent magnitude in the single. The single yarn most suitable for doubling is that which possesses the most generous combination of the following characteristics:-Minimum of fluctuation in strength, diameter, count, and twist; sound fibres, well ordered, twisted to the requirements of the folding twist; and freedom from all kinds of faulty places. The utility of the yarn depends upon the exactness of the single twist and the quality. Quality is represented numerically by the measure of strength in relation to the quantity of fibres and twist present in the yarn. Therefore, strength in relation to the twist and count reveals by its variations the measure of stability which the yarn attains. Low twist, in the singles, consistent with the needs of the various doubled yarns, produces the most desirable effects generally. Low twist singles are not popular, and hence the minimum twist limit is avoided by a considerable margin on the ground of unreliability or difficulty in working. It is possible, by modifying the ordinary system of presenting the yarn in doubling so that the twist flows freely to the point of introduction, to cause soft twisted singles to work without manifesting the troubles in question.

The matters of which I have spoken may appear very simple, but they are of decided importance. I have treated them as being things which are not fully understood, because, so far as I am aware, thoy have not been raised previously. I trust that I may not have failed to present my views in a manner which will allow of useful application in the work of both spinning and doubling.

\section{Discussion.}

In opening the proceedings, the Charrman stated that this was the first meeting of the Textile Institute in Stockport. Stockport was a large industrial centre, with ten to twelve thousand operatives engaged in doubling. It was the chief town in the trade.

At the conclusion of the lecture, Mr. Higson invited discussion and questions.

Mr. H. P. GREG said it had given him great pleasure to listen to the lecture. He would suggest that $\mathrm{Mr}$. Winterbottom should give practical light on the testing of yarns-especially single yarns. The ordinary lea test was unsatisfuctory. He would like to ask $\mathrm{Mr}$.
Winterbottom whether he thought a mechanica] method of putting in twist would give a more regular effect in doubling. American frames were run by gearing. Was any advantage achieved by this, rather than by driving the spindles by bands or frictionally ?

Mr. Wintenвotrom said that positive driving had been attempted in doubling and spinning. A positive machine was used in the flax, wool, and coarser doubled yarn trades. It was a modified fly frame with positive driving and twisting. The yarn made on every spindle in the frame contained the same number of twists. If positive driving were adopted with ordinary doubling frames, some form of clutch would be necessary and more attention would be required in that case. The flexible drive was all right when the bands were the best of the kind. The conditions must be as uniform as possible. As regards appliances for testing, the lea tester break represented the yarn inadequately. The breakage of a very few threads caused the finger of the machine to cease moving. The strength of the weakest threads determined the utility of yarn, and, therefore, the nearer the minimum was to mean and the maximum, the better the yarn. Most of the single thread testers were too slow, the Moscrop being an exception. All break test figures should be corrected for deviations in count and twist. In the correction of the results of strength tests, it was recognised that deviation in count-according to the various tables of strength of yarn-affected strength in one of the following orders : (1) the inverse proportion ; (2) square root of count ; (3) square root \pm a deviation. The last was more nearly correct than the other two. Assuming that the yarn followed any one of these laws, 80's yarn in com. parison with 79's (reputed 80 's) had a wealmess in proportion to the fibres contained. The strength must, therefore, be adjusted to the extent of the deviation in count. Then, in regard to deviation in twist, the strength must be corrected to the extent of from square root of 80 to square root of 79 .

Mr. Greg : Tyre yarns, in America, are made with one doubling, and in England with two doublingsthat is, cabled yarns. What are the advantages of one doubling, or two doublings, as applied to tyre yarns ?

Mr. Hiason : In 6-cord yarn is there any advantage in doubling three ends (twofold) together, instead of six ends separately?

Mr. Winterbottom : As regard Mr. Greg's question, multiple or cabled yarns are stronger at the finish, but less elastic. The Americans make simple folded yarns to obtain greater resiliency, whereas we make cabled yarns. In cases, it is done because less resiliency and greater strength per unit of fibres is required. Regarding Mr. Higson's query, for strength types three twofolds yield higher in strength, wear, and stability as compared with sixfold (singles); there is also the difference in controlling tension. The aim in doubling should be to reduce the twist in the fibres to the fewest practicable.

On the motion of Mr. Grea, seconded by Mr. A. Dowson, and supported by Mr. T. FLETCher RoBINSON (Chairman of the Lancashire Section), a hearty vote of thanks was accorded the Lecturer and the Chairman. 